\title{
BMJ Open Protocol for a longitudinal study to evaluate the use of tenofovir-based PrEP for safer conception and pregnancy among women in South Africa
}

Lynn T Matthews, ${ }^{01,2}$ Manjeetha Jaggernath, ${ }^{3}$ Yolandie Kriel, ${ }^{3}$ Patricia M Smith, ${ }^{1}$ Kasey O'Neil, ${ }^{4}$ Jessica E Haberer, ${ }^{4}$ Craig Hendrix, ${ }^{5}$ Jared M Baeten, ${ }^{6}$ Norma C Ware, ${ }^{7}$ Kathleen Wirth, ${ }^{8}$ Christina Psaros, ${ }^{9}$ David R Bangsberg, ${ }^{10}$ Jennifer A Smit ${ }^{3}$

To cite: Matthews LT, Jaggernath M, Kriel Y, et al. Protocol for a longitudinal study to evaluate the use of tenofovirbased PrEP for safer conception and pregnancy among women in South Africa. BMJ Open 2019;9:e027227. doi:10.1136/ bmjopen-2018-027227

- Prepublication history and additional material for this paper are available online. To view these files, please visit the journal online (http://dx.doi. org/10.1136/bmjopen-2018027227).

Received 13 October 2018 Revised 6 March 2019 Accepted 12 June 2019

Check for updates

(C) Author(s) (or their employer(s)) 2019. Re-use permitted under CC BY-NC. No commercial re-use. See rights and permissions. Published by BMJ.

For numbered affiliations see end of article.

Correspondence to

Dr Lynn T Matthews;

lynnmatthews@uabmc.edu

\section{ABSTRACT}

Introduction Women who choose to conceive a baby with a partner living with HIV or a partner whose HIV serostatus is unknown in HIV-endemic settings need prevention strategies to mitigate HIV acquisition during conception and pregnancy.

Methods and analysis We are conducting a singlearm longitudinal study offering oral tenofovirdisoproxil fumarate/emtricitabine (TDF/FTC) as pre-exposure prophylaxis (PrEP) for periconception use to $350 \mathrm{HIV}$ uninfected women in KwaZulu-Natal, South Africa. PrEP is offered as part of woman-centred safer conception programme that promotes couples-based HIV counselling and testing, antiretroviral therapy for partners who are HIV-infected, treatment for sexually transmitted infections and safer conception strategies, such as limiting condomless sex to peak fertility. We enrol HIV-uninfected women who are not currently pregnant, in a stable relationship ( $\geq 6$ months) with a partner living with HIV or of unknown serostatus, and personal or partner plans for pregnancy in the next 12 months. We follow enrolled women for 12 months. Women who become pregnant are followed through pregnancy outcome, independent of their decisions regarding PrEP use. The primary objective of the study is to evaluate the uptake of and adherence to PrEP during the periconception period and pregnancy. Secondary outcomes include the uptake of other safer conception strategies. We also measure clinical outcomes including HIV seroconversion rates and pregnancy and infant outcomes. Finally, we will explore conduct and evaluate qualitative interviews in 25 participants to further inform our conceptual framework for periconception PrEP uptake and adherence among HIV-exposed women in South Africa.

Ethics and dissemination The protocol has been approved by the Human Research Ethics Committee at the University of the Witwatersrand (Johannesburg, South Africa) and the Institutional Review Board of Partners Healthcare (Boston, Massachusetts, USA). Study findings will be made available to interested participants. Results will be presented to local health officials and stakeholders at meetings. Investigators will share the results at meetings and in manuscripts. De-identified quantitative data will be made available.

\section{Strengths and limitations of this study}

- This study will objectively measure uptake of and adherence to pre-exposure prophylaxis (PrEP) during conception and pregnancy.

- This study will evaluate the use of PrEP during pregnancy in South Africa where tenofovir disoproxil fumarate/emtricitabine is currently contraindicated in pregnancy.

- Enrolling women who may be exposed to HIV during conception attempts, but without requiring PrEP use, allows for evaluation of uptake.

- Enrolling women independent of their partners allows for evaluation of adherence outside of mutually disclosed HIV-serodifferent partnerships.

- We will not test male partners' HIV-serostatus or necessarily hear their perspective directly as men are not enrolled.

Trial registration number The protocol is registered with the South African Health Products Regulatory Agency (SAHPRA, formerly known as the Medicine Controls Council, MCC\#20170131) and ClinicalTrials.gov (NCT03194308); Pre-results.

\section{INTRODUCTION}

In HIV-endemic settings, many HIV-uninfected women choose to conceive a baby with a partner living with HIV or whose serostatus is not known. ${ }^{1-4}$ For a woman who cannot depend on a partner to test, initiate and adhere to ART, and suppress HIV RNA, condomless sex puts her at risk of acquiring HIV and increases the risk of perinatal transmission to her child. ${ }^{5}$ In South Africa, HIV incidence has declined since 2012, but women of reproductive age have the highest incidence at $0.93 \% .{ }^{6}$ Even with the roll-out of test and treat antiretroviral therapy (ART) policies in South Africa, in some settings, 
HIV incidence among women continues to rise even as incidence declines among men, ${ }^{7}$ reflecting ongoing challenges to supporting men living with HIV to engage and remain in care. ${ }^{8-12}$

Preventing HIV infection among women of reproductive age is the first pillar of reducing perinatal transmission. ${ }^{13}$ Although pregnant women living with HIV are a major focus of services to prevent perinatal transmission, HIV prevention prior to pregnancy receives relatively little attention. The contribution of intended conception to incident HIV infection is important in many HIV-endemic settings given the importance of having children, a lack of programmes addressing periconception transmission and high HIV prevalence. ${ }^{14}$

Daily, oral tenofovir disoproxil fumarate/emtricitabine (TDF/FTC) for pre-exposure prophylaxis (PrEP) dramatically reduces a woman's risk of HIV acquisition and may be an important female-controlled option for reducing the risk of periconception HIV acquisition. ${ }^{15}{ }^{16}$ Evidence suggests it is safe to use during pregnancy. ${ }^{17}$ Understanding whether daily, oral PrEP is feasible for uninfected women seeking pregnancy is critical to reducing HIV incidence among women and their children.

Placebo-controlled trials identified adherence as a major challenge to long-term PrEP use among women. ${ }^{18}$ However, women are eager for prevention strategies that allow for conception, ${ }^{19-21}$ and we hypothesise that adherence to a proven prevention strategy, for a limited time with the motivation to have a healthy child, will confer the drug levels required to prevent HIV transmission. Supporting our hypothesis is the finding that women had a high adherence prior to conception in clinical trials and demonstration projects of PrEP. ${ }^{192-24}$

Our conceptual framework is based on an ecological framework published by van der Straten $e t$ al and adapted based on our periconception risk behaviour conceptual framework. ${ }^{25-27}$ This framework assumes that uninfected women who want to have a child with an infected or unknown serostatus partner will experience individual, couple and community-level factors that influence PrEP initiation and adherence behaviour. At the individual level, she may have excellent knowledge of safer conception strategies, be optimistic about PrEP efficacy and perceive herself to be at high risk for acquiring HIV. However, on the dyadic level, she and her partner may not communicate or trust one another, making it hard for her to adhere to daily medication. If she feels that childlessness and/or being HIV-infected is highly stigmatised in the community, then she may be more motivated to participate in periconception PrEP. Our mixed methods data will allow us to develop this framework to inform interventions for women seeking HIV-prevention during periconception and pregnancy.

This manuscript describes the ZINK protocol. 'Zivikele ngaphambi kokukhulelwa' means, 'Protecting yourself before pregnancy' in isiZulu. This project will inform whether daily, oral PrEP is a feasible component of comprehensive HIV prevention for South African women who intend to conceive a baby and may be exposed to HIV. Given the repercussions of acquiring HIV during conception and pregnancy, periconception PrEP is an important step towards supporting women to safely meet reproductive goals.

\section{METHODS AND ANALYSIS \\ Study design}

The Zivikele ngaphambi kokukhulelwa (ZINK) or Protecting yourself before pregnancy study is a single-arm longitudinal study offering safer conception counselling to HIV-exposed women in Durban, South Africa. The safer conception package emphasises the importance of couples-based HIV counselling and testing (CHCT), ART for the infected partner, treatment for sexually transmitted infections (STIs), and safer conception strategies, such as limiting sex without condoms to peak fertility. We also offer TDF/FTC as PrEP. Study participants are followed for a period of 12 months. Those with pregnancy during the 12-month follow-up are counselled about HIV risk-reduction during pregnancy, offered PrEP and followed through to pregnancy outcome (regardless of PrEP use).

\section{Population}

ZINK participants are women aged 18-35 years who are HIV-negative with personal or partner plans for pregnancy with an infected or unknown serostatus partner. Women are recruited in Durban, KwaZulu-Natal, South Africa where nearly $40 \%$ of women who attend antenatal clinics test positive for HIV infection. ${ }^{28}$ PrEP is currently contraindicated for use by pregnant women in South Africa, due to local concerns regarding safety outlined by the South African Health Products Regulatory Agency (SAHPRA, formerly known as the MCC). ${ }^{29}$ Due to challenges recruiting HIV-serodifferent couples in this setting, we recruit women as individuals ${ }^{30}$; however, women are invited to bring their partners to the study site for counselling and information.

Recruitment for this study is through fieldwork teams reaching out directly to women at local Department of Health primary healthcare clinics within the eThekwini District, gathering spots near the research site (such as hair salons and service queues), and through word-ofmouth promotion from enrolled participants and others who know of the study. We are also planning community events in consultation with our affiliate Community Advisory Board (CAB). Recruitment began in November 2017 and is expected to last 30 months.

\section{Study aims and outcomes}

The primary aims of the study are to: (1) determine PrEP uptake and adherence among all enrolled participants (by self-report, electronic monitoring, plasma and blood samples), (2) determine PrEP adherence among women who take PrEP during the periconception period and pregnancy and (3) explore adherence facilitators and barriers during periconception and pregnancy to further 
inform our conceptual framework for periconception PrEP uptake and adherence. ${ }^{27}$ Additional outcomes of interest include uptake of safer conception strategies including CHCT, ART for the infected partner and contraception uptake for those who decide not to conceive after careful consideration. We also measure clinical outcomes including HIV seroconversion rates, pregnancy outcomes (eg, miscarriage, stillbirth and live birth) and infant outcomes (eg, weight, length and observed congenital anomalies).

\section{Eligibility criteria}

Study inclusion and exclusion criteria are presented in box 1 and include (via self-report) being woman; between ages 18 and 35 years; reporting an HIV-seropositive or unknown HIV serostatus partner; not on long-acting contraception (IUD (Intrauterine Device), injectable or implanted contraception); and personal or partner plans to have a child within the next year. Interested women are screened for infertility by history ${ }^{31}$ and participants who fail to meet the fertility criteria are considered ineligible. Women who are eligible based on the self-report data complete biological screening. They are eligible to enrol in the study if they test negative for pregnancy and HIV and are not co-enrolled (checked via fingerprint ${ }^{32}$ ) in other research studies that may conflict with our procedures (eg, vaccine trials, PrEP projects and so on).

\section{Informed consent procedures}

Interested women are asked to complete a brief verbal informed consent prior to completing the screening questions (box 1). Women are eligible after completing the screening questions will be invited to complete an initial written informed consent where the secondary, biological screening process will be explained (box 1). If women are eligible for study enrolment, they will be invited to complete an in-depth informed consent process for study enrolment and participation.

The Research Assistants will use informed consent documents available in both English and isiZulu as a guide to discuss all the content in the informed consent with the potential participant. The consent forms will be reviewed orally by a study representative and the participant will be encouraged to ask detailed questions. Women who meet study inclusion/exclusion criteria and complete the informed consent process and give signed consent will then be enrolled.

\section{Study activities}

Study activities and corresponding visit schedules are shown in table 1 and further explained below.

\section{Counselling}

At the enrolment visit, women are offered a package of safer conception counselling based on South African guidelines. ${ }^{33}$ These guidelines recommend that HIV-uninfected women who want to conceive a child with a partner living with HIV should encourage partner testing and serostatus disclosure within the partnership, encourage partner to initiate ART, delay sex without condoms until he achieves HIV RNA suppression or is on ART for 6 months, consider contraception to delay pregnancy until safer conception strategies implemented, limit condomless sex to peak fertility, and/or consider sperm washing, donor sperm and adoption as alternatives. In this study, safer conception counselling occurs at baseline and at each visit thereafter for non-pregnant women. Comprehensive counselling is performed by trained study counsellors in a group format, with the option of one-on-one counselling sessions based on participant preference and feasibility of convening a group. We developed and pilot tested locally relevant tools and visual graphics to communicate key concepts in prior studies ${ }^{3435}$ (see figure 1A-C for key concepts).

Women who initiate PrEP participate in brief adherence counselling and support sessions at each visit to discuss barriers to and promoters of adherence. A group format is pursued given interventions that target social networks and strengthen group ties have been used to empower marginalised groups and to effect health-related behaviour change related to HIV prevention. ${ }^{36-38}$

\section{Box 1 Inclusion and exclusion criteria}

\section{Verbal screening inclusion criteria}

- Female.

- Aged between 18 and 35 years.

- Sexually active.

- Likely to be fertile based on responses to reproductive history assessment.

- Personal or partner desire to have a child in the next year.

- With a stable (together for at least 6 months) partner, she reports as HIV-infected or HIV-serostatus unknown (if $>1$ desired pregnancy partner, we will ask her to identify the most likely pregnancy partner based on her own assessment of sexual frequency, fertility and so on).

- Able to participate in the informed consent process.

- Fluent in English or isiZulu.

\section{Secondary inclusion after informed consent}

- Co-enrolment in studies which may conflict with participation in this study.

- HIV-negative (rapid testing).

- Not pregnant (by urine beta-hCG testing).

\section{Exclusion criteria}

- HIV positive.

- Pregnant.

- No desire to have a child in the next 12 months.

- Living at or planning to relocate to a location incompatible with study participation in the next year.

- Active drug or alcohol use that, in the opinion of the research study team, would interfere with adherence to study requirements.

- Active illness requiring systemic treatment and/or hospitalisation within 30 days prior to study entry that in the opinion of the research study team, might otherwise interfere with adherence to study requirements.

- Inability to adhere to the study schedule and/or study procedures.

beta-hCG, beta-human chorionic gonadotropin. 
Table 1 Study procedures for non-pregnant and pregnant participants

\begin{tabular}{|c|c|c|c|c|c|c|}
\hline Procedures & Enrolment & M1 & M3 & M6 & M9 & M12 \\
\hline \multicolumn{7}{|l|}{ Counselling } \\
\hline \multicolumn{7}{|l|}{ Safer conception counselling } \\
\hline \multicolumn{7}{|l|}{ Adherence support if taking PrEP } \\
\hline \multicolumn{7}{|l|}{ Laboratory } \\
\hline \multicolumn{7}{|l|}{ Urine pregnancy test ${ }^{\star}$} \\
\hline \multicolumn{7}{|l|}{ HIV rapid test } \\
\hline \multicolumn{7}{|l|}{ STI symptom screen } \\
\hline \multicolumn{7}{|l|}{ HBV serology if initiating PrEP } \\
\hline \multicolumn{7}{|l|}{ Creatinine if initiating PrEP and quarterly while on PrEP } \\
\hline \multicolumn{7}{|l|}{ Blood sample for tenofovir levels if on PrEP } \\
\hline \multicolumn{7}{|l|}{ Sociobehavioural data } \\
\hline \multicolumn{7}{|l|}{ Sociodemographic information } \\
\hline \multicolumn{7}{|l|}{ Brief questionnaire } \\
\hline \multicolumn{7}{|l|}{ Full questionnaire } \\
\hline \multicolumn{7}{|l|}{ Weekly SMS sexual behaviour data } \\
\hline MEMS cap adherence data and questionnaire if on PrEP & & & & & & \\
\hline In-depth interviews with a subset of women & & & & & & \\
\hline
\end{tabular}

*Once pregnancy is diagnosed, women reconsent to participate in the study and are seen monthly for HIV testing and safety monitoring. They maintain a schedule for quarterly counselling and adherence support. A postpregnancy follow-up visit will assess pregnancy and infant outcomes.

HBV, hepatitis B virus; MEMS, Medication Event Monitoring System; PrEP, pre-exposure prophylaxis; STI, sexually transmitted infection.

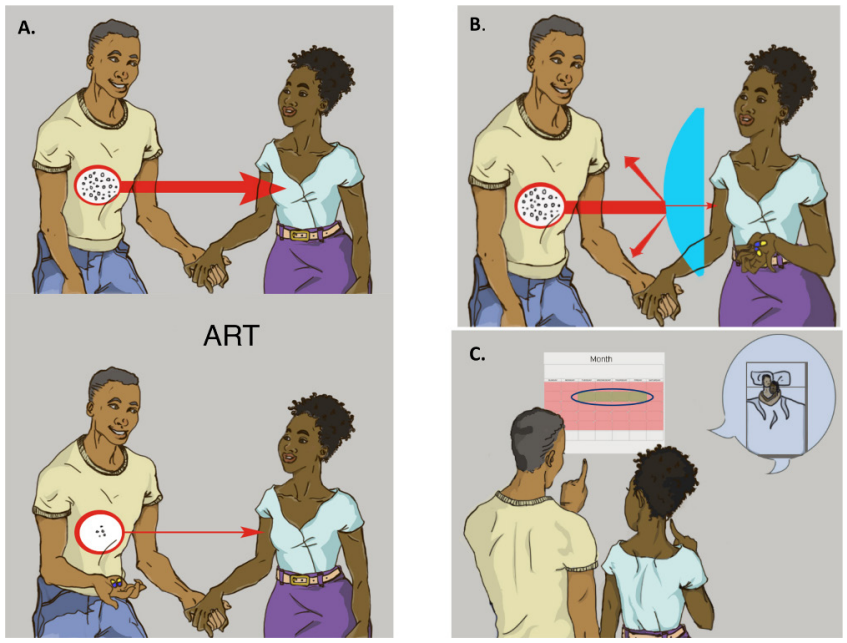

Figure 1 Locally-relevant images developed to convey key safer conception strategies in the safer conception counselling sessions. (A) Treatment as prevention. (Top panel) The man is not taking ART, has a high viral load and there is a high risk of transmitting to his partner. (Down panel) The man is taking ART, has a suppressed viral load and the risk of transmitting to his partner is minimized. (B) PrEP. The man is not taking ART, has a high viral load and there is a high risk of transmitting to his partner. In (B), the woman is taking PrEP which protects her from acquiring HIV. (C) Timing condomless sex to peak fertility. In this image, the couple is evaluating a calendar to time condomless sex to peak fertility. ART, antiretroviral therapy; PrEP, pre-exposure prophylaxis.
Participants will also have the option of participating in one-on-one counselling sessions based on their preference and schedule.

\section{Laboratory}

Participants complete beta-HCG urine pregnancy testing, individual HIV counselling and testing (HCT), and syndromic screening for STIs at each study visit. Study staff will follow-up with participants who seroconvert to promote linkage to care and conduct genotyping. Participants with a positive pregnancy test are referred to antenatal care and those with STI symptoms are referred to a local clinic for treatment.

Women who choose to initiate PrEP undergo blood testing for renal function (creatinine) and for hepatitis B infection. Women with abnormal renal function (serum creatinine $>89 \mu \mathrm{mol} / \mathrm{L}$ and estimated glomerular filtration rate $($ eGFR $)<60 \mathrm{~mL} / \mathrm{min}$ or serum creatinine $>85$ $\mu \mathrm{mol} / \mathrm{L}$ for pregnant women) or active hepatitis B infection (hepatitis B surface antigen positive) are instructed to stop PrEP.

Renal function testing is repeated quarterly in accordance with CDC and WHO guidelines for safety monitoring. PrEP can be stopped at any point should there be the abnormal renal function as per the above definition. PrEP can be re-started if serum creatinine and/or eGFR levels return to normal. Pregnant women who decide to continue PrEP during pregnancy are retested for hepatitis 
Table 2 Weekly SMS sexual behaviour survey delivered to their mobile phone in their language of choice (English or isiZulu)

\begin{tabular}{|c|c|}
\hline Question & Response options \\
\hline $\begin{array}{l}\text { Did you have sex in } \\
\text { the past } 7 \text { days? }\end{array}$ & $\begin{array}{l}\text { 1. Yes } \\
\text { 2. No } \rightarrow \text { stop questionnaire } \\
\text { 3. I prefer not to answer }\end{array}$ \\
\hline Which partner(s)? & $\begin{array}{l}\text { 1. Healthy baby partner } \\
\text { 2. Other partner(s) } \\
\text { 3. Healthy baby and other partners } \\
\text { 4. I prefer not to answer }\end{array}$ \\
\hline I used a condom... & $\begin{array}{l}\text { 1. Every time } \\
\text { 2. Some of the time } \\
\text { 3. Never } \\
\text { 4. I prefer not to answer }\end{array}$ \\
\hline $\begin{array}{l}\text { Were you in your } \\
\text { fertile period? }\end{array}$ & $\begin{array}{l}\text { 1. Yes } \\
\text { 2. No } \\
\text { 3. Do not know } \\
\text { 4. I prefer not to answer }\end{array}$ \\
\hline
\end{tabular}

$\mathrm{B}$ and kidney function at the time of their positive pregnancy test. All women who chose PrEP during pregnancy undergo renal function testing every 3 months until pregnancy outcome.

\section{Questionnaires}

At month 0,6 and 12, a full questionnaire is administered to assess constructs within our conceptual framework for periconception risk behaviour (eg, risk perception, reproductive autonomy and HIV stigma) ${ }^{27}$ (online supplementary appendix 1). Constructs are measured using instruments validated in this setting. Questionnaires are administered via face-to-face interviews with a trained research assistant fluent in English and isiZulu, which is the dominant local language. At month 3 and 9, a brief questionnaire is administered to explore safer conception behaviours including self-reported partner HIV status, delay of sex without condoms until the partner on ART is virologically suppressed, and access to additional services such as sperm washing.

At the enrolment visit, participants are asked to sign up for a weekly four-question SMS sexual behaviour survey delivered to their mobile phone. The questions and response options are listed in table 2. These password-protected SMS-reported sexual behaviour data will allow for the measurement of adherence relative to HIV exposure. $^{39}$

\section{PrEP use}

Participants who choose to use PrEP are offered oral, daily TDF/FTC PrEP (provided by Gilead) according to US CDC guidelines for periconception PrEP and WHO Guidelines for PrEP use among those at substantial risk for acquiring HIV. ${ }^{40}{ }^{41}$ Women are counselled to use PrEP for 7 days (as per WHO 2016 guidance) before engaging in sex without condoms or other backup protection.
Women are counselled to continue PrEP for at least 28 days after the last HIV exposure.

PrEP uptake is defined as the proportion of enrolled women who collect at least 1 month's supply of PrEP within the first 12 months of study participation. Women may choose to initiate/discontinue PrEP at any time during the periconception period. At each quarterly visit, a 90-day prescription is given to participants (non-pregnant and pregnant) who chose to use PrEP. Women who become pregnant may choose to continue or initiate PrEP, with a plan to continue through to pregnancy outcome or until her risk is resolved.

Participants taking PrEP are provided AARDEX Medication Event Monitoring System (MEMS), a bottle cap with an electronic chip that clocks bottle openings. At each study visit, MEMS data will be downloaded by study staff and a questionnaire will be completed to evaluate for self-reported adherence. We will evaluate adherence to PrEP through plasma tenofovir (TFV) and diphosphorylated tenofovir (TFV-DP) levels in dried blood spots. Samples will be collected 3 months after PrEP initiation and at quarterly visits thereafter. Detectable $(>0.31 \mathrm{ng} /$ $\mathrm{mL}$ ) TFV indicates at least one dose in the prior 7 days, $>10 \mathrm{ng} / \mathrm{mL}$ indicates dosing in the last 2-3 days and $>40 \mathrm{ng} / \mathrm{mL}$ indicates dosing in the last 24 hours and is associated with HIV protective efficacy. ${ }^{42}{ }^{43}$ We anticipate performing the DBS TNF-DP assay on 20 percent of the total number of samples. The TNF-DP data will give a sense of longer-term adherence and may help assess the 'white coat' effect in this sample. ${ }^{44-46}$ Adherence questions at each visit and sexual behaviour data will be used to evaluate for adherence relative to HIV exposure or 'prevention-effective' adherence. ${ }^{18}$

\section{In-depth qualitative individual interviews}

In-depth qualitative individual interviews will be conducted with a subset of up to 25 women based on a 'purposeful sampling' framework. ${ }^{47}$ Trained research assistants will conduct initial (up to 6 months after enrolment) and exit qualitative interviews to explore factors affecting PrEP uptake and adherence during periconception and pregnancy. Pregnant and non-pregnant women will be selected from three groups of roughly equal distribution including (1) those who did not choose PrEP as part of a safer conception strategy, (2) those who chose PrEP and/or have detectable plasma TFV and/ or $\geq 80 \%$ MEMS adherence and (3) those who chose PrEP but have undetectable plasma TFV and/or $<80 \%$ MEMS adherence. Additionally, we will follow women over the course of the study and offer repeat event-triggered qualitative interviews after major life events such as pregnancy, major change in partnership (eg, participation in CHCT, disclosure and separation from current partner) or change in PrEP use. Based on our conceptual framework, we assume that uninfected women who want to have a child with an infected or unknown serostatus partner will experience individual, couple and community-level factors that influence PrEP initiation and adherence 
behaviour. At the individual level, she may have excellent knowledge of safer conception strategies, be optimistic about PrEP efficacy and perceive herself to be at high risk for acquiring HIV. However, on the dyadic level, she and her partner may not communicate or trust one another, making it hard for her to adhere to daily medication. If she feels that childlessness and/or being HIV-infected is highly stigmatised in the community, she may be more motivated to participate in periconception PrEP (online supplementary appendix 2.) By collecting qualitative data as well as quantitative covariates that reflect the constructs of this framework, our mixed methods data will allow us to adapt this framework as appropriate to inform interventions for women seeking HIV prevention during periconception and pregnancy.

\section{Pregnant participants}

Participants who become pregnant during the study have the option to continue participating in the study (per GCP guidelines a woman who becomes pregnant must reconsent to participate in this study). If a woman has not chosen PrEP, then she can choose to start. If she has already chosen PrEP, then she can choose whether or not to continue. Pregnant participants are referred to their local clinic for antenatal follow-up and care. Pregnant participants receive counselling regarding the risks and benefits of using PrEP during pregnancy. Women who plan to reduce HIV exposure without PrEP (eg, partner on ART with suppressed viral load, routine condom use and HIV-negative partner) will be encouraged to pursue these methods of risk reduction. Women who cannot confidently implement strategies to reduce HIV acquisition risk will be supported to start or to continue to use PrEP, during pregnancy. This approach is consistent with US CDC and WHO guidelines for PrEP use,${ }^{40}{ }^{41}$ but is not part of South African guidelines where TDF/FTC as PrEP is contraindicated for use in pregnancy given safety concerns. ${ }^{29}$ At the conclusion of pregnancy, women will be asked about the pregnancy outcome and their clinical charts will be reviewed to collect additional relevant data, such as live birth, stillbirth or miscarriage.

Women who become pregnant during the first 12 months of follow-up will be asked to bring their child in for evaluation by the study doctor at their final visit (within 3 months of pregnancy end), to document birth date, outcomes and conduct an examination to evaluate for any congenital abnormalities. The study team will also ask permission to contact the labour and delivery unit and/or paediatrician for additional details. For women who do not return for this visit, loss to follow-up procedures will be followed including contacting the participant, home visits, and reviews of the medical record at the facility where she sought antenatal care and/or planned to deliver. Permission to conduct off-site visits and access healthcare records will be obtained during the pregnancy consenting process.
Women will be reimbursed 250 South African Rand ( 20 US\$) per scheduled visit consistent with South Africa's local guidance.

\section{Patient and public involvement}

Years of mixed methods research with men and women affected by HIV informed this proposal. ${ }^{114} 19284748$ In addition, the study team consulted with MatCH Research Unit's affiliate $\mathrm{CAB}$ who informed our understanding and appreciation of community members' personal and familial experiences related to a woman's choice to have a child with a partner who is living with HIV or with an unknown serostatus partner. They also aided in our understanding of women's need for, and the complexities related to, informed and supported prevention strategies to reduce periconception HIV acquisition risk. The CAB advised the research team on appropriate recruitment venues, recommended that study team members present the study at community council meetings, and posted study flyers at clinics and community gathering spots.

In addition to recruitment efforts by the study team and the $\mathrm{CAB}$, the participants themselves are involved in ongoing word-of-mouth recruitment. The results of the study will be disseminated to participants at community gatherings organised by the study team in conjunction with $\mathrm{CAB}$ members.

\section{Data analysis}

Aims and outcomes

\section{PrEP uptake}

We will calculate the proportion (and 95\% CI) of women who initiate PrEP. PrEP uptake is defined as the proportion of enrolled women who collect at least 1 month's supply of PrEP within the first 12 months of study participation. We will describe and compare baseline characteristics among those initiating PrEP versus those not initiating PrEP and test for differences in the distribution of characteristics in the two groups using the t-test or Wilcoxon rank sum test, as appropriate, for continuous variables and $\mathrm{X}^{2}$ or Fisher exact test as appropriate for categorical variables. Log-binomial regression will be used to model the association between characteristics of interest (eg, partner HIV serostatus) and PrEP initiation and to calculate unadjusted risk ratios and 95\% CIs. Covariates that are marginally associated with PrEP initiation at $\mathrm{p}<0.1$ will be considered for inclusion in multivariable models and adjusted ORs and 95\% CIs will be calculated.

\section{PrEP adherence}

Adherence implementation will be defined as the number of MEMS cap openings divided by the total number of doses prescribed over the study interval in the periconception period. Adherence persistence refers to the duration of PrEP use, defined as the time (in weeks) from PrEP initiation to either the first interval period of at least 1 week with no openings during a period of intended overage, undetectable plasma TFV or study completion. We will also evaluate this in the context of PrEP need 
(eg, if the woman remains at risk for HIV with respect to sexual behaviour, partner serostatus and partner viral suppression).$^{18}$

The primary adherence outcome is defined among PrEP initiators as a protective $(>40 \mathrm{ng} / \mathrm{mL})$ plasma TFV concentration, measured quarterly (binary outcome). Secondary adherence outcomes are based on electronic monitoring with MEMS caps. We will determine the mean and median number of pills taken as well as patterns of adherence (gaps). We will consider MEMS adherence as a binary outcome defined as taking $\geq 80 \%$ of prescribed doses based on biologic plausibility and prior studies. ${ }^{48-53}$ We will also explore adherence as a continuous outcome and will determine longitudinal trends in adherence through monthly assessments of median (IQR) adherence and adherence gaps of at least 72 hours and PrEP use in the context of HIV-exposure.

For the prevalence of PrEP adherence (binary), we will use a log-binomial regression model (intercept term only) with generalised estimating equations (GEE) in the setting of repeated measurements. We will extend this model to include baseline and time-updated characteristics of interest to estimate unadjusted relative risks and $95 \%$ CI. For continuous adherence outcomes using MEMS cap data, we will describe the distribution of adherence and compare mean and median adherence levels across the full periconception period in those exposed versus not exposed to baseline and time-updated factors of interest.

\section{Additional outcomes}

We will also describe, as additional outcomes, pregnancy incidence, HIV seroconversion (and compare rates among those taking and adherent to PrEP with those who do not take or adhere to PrEP) and all pregnancy outcomes. The samples size will be too small to carry out adjusted analyses, but bivariate associations will inform factors that affect the uptake of and adherence to PrEP. We will also evaluate the use of other safer conceptions strategies (eg, CHCT, delay of sex without condoms until a partner tests for HIV and is either HIV-uninfected or HIV-positive, accessing ART and suppressed, and access of additional services such as sperm washing.)

Longitudinal quantitative data and serial in-depth interviews with initially high-adhering and low-adhering participants as well as participants who do not initiate PrEP will explore barriers to and promoters of PrEP uptake and adherence while informing our conceptual framework. Grounded theory methods will be used to analyse qualitative interview data. These methods lay out a systematic set of inductive procedures for developing categories of information, organising data in terms of these categories through coding, connecting the categories and deriving from these connections an empirically grounded theory. We will also use structural equation modelling to examine the associations and putative causal pathways among the hypothesised determinants of adherence as described in the conceptual framework (eg, individual level motivations such as parenthood motivation, dyadic factors such as relationship power, and community-level factors such as functional support).

\section{Clinical outcomes relating to PrEP safety}

We will describe clinical outcomes relating to the safety of PrEP use in pregnancy including pregnancy outcomes (miscarriage, stillbirth and live birth) and infant outcomes inclusive of weight, length, head circumference and any noted congenital anomalies. Incidence rates will be compared across women exposed/not exposed to TDF/ FTC as PrEP and also compared with population norms.

\section{Power and sample size considerations}

We will calculate the proportion of women who initiate PrEP over a 12-month period. A target sample size of 350 women produces a $95 \%$ CI width of $9.9 \%$ when the percent of women initiating PrEP is $70 \%$ and a $95 \%$ CI width of $10.5 \%$ when the percent of women initiating PrEP is $50 \%$ (eg, $70 \%$ (95\% CI $65 \%$ to $75 \%$ )), providing reasonable precision for an estimate of PrEP uptake.

Among women who initiate PrEP, we will calculate the proportion of women adherent to at least $80 \%$ of prescribed doses in the past month. For this and other binary adherence outcomes, a target sample size of 245 women initiating PrEP produces a 95\% CI width of $10.4 \%$ when the percent of women who are high adherers to PrEP is $80 \%$. For continuous adherence outcomes, this sample size produces a two-sided $95 \%$ CI with a distance from the mean to the limits that is equal to $0.126 \mathrm{SD}$ when the estimated $\mathrm{SD}$ is 1.000 .

\section{Lost to follow-up data}

Data from women lost to follow-up will be censored at last contact. In addition, to adjust for potential bias due to loss to follow-up among pregnant women, we will conduct a sensitivity analysis using the inverse probability of censoring weighting. ${ }^{545}$

\section{Ethics and dissemination}

This study team has considered the ethical issues in line with ICH-GCP (The International Conference on Harmonization of Technical Requirements for Registration of Pharmaceuticals for Human Use - Good Clinical Practice) guidelines. Given normative guidance for PrEP use in pregnancy in South Africa, the DSMB will review safety data including adverse infant outcomes (preterm birth ( $<37$ weeks gestation), stillbirths, low birth weight $(<2500 \mathrm{~g})$ and observed congenital anomalies). Study clinicians will carefully monitor adverse events in women choosing PrEP and pregnant women at all scheduled study visits. All data on pregnancy use and TDF/FTC exposure are reported to the Antiretrovirals in Pregnancy Registry (http://www.apregistry.com/). Study results will be disseminated at national and international conferences and in peer-reviewed journals. The trial findings may also be made available to participants collectively, should participants agree. 
The protocol has been approved by the Human Research Ethics Committee at the University of the Witwatersrand (Pretoria, South Africa) and the Institutional Review Board of Partners Healthcare (Boston, Massachusetts, USA). The protocol is registered with the South African Health Products Regulatory Agency (SAHPRA), MCC\#20170131) and at ClinicalTrials.gov (NCT03194308).

Results will be presented to local health officials and stakeholders at meetings. Investigators will share the results at meetings and in manuscripts. De-identified quantitative data will be made available. Qualitative data will not be made available due to challenges to redacting identifiers in a potentially-stigmatised population of HIV-exposed women planning pregnancy. ${ }^{56}$ We will aim to follow STROBE guidelines in the presentation of results.

\section{CONCLUSION}

For women who cannot depend on a partner to test, initiate and adhere to ART, and suppress HIV-RNA, condomless sex puts her at risk of acquiring HIV and increases the risk of perinatal transmission to her child. This study will provide important objective information regarding women's choices and use of PrEP and other safer conception strategies in this context. We will also collect additional safety data regarding TDF/FTC-exposed pregnancies.

\section{Trial status}

The study is currently recruiting participants.

\section{Author affiliations}

${ }^{1}$ Department of Medicine, Division of Infectious Diseases, University of Alabama at Birmingham (UAB), Birmingham, Alabama, USA

${ }^{2}$ Department of Medicine, Division of Infectious Diseases, Massachusetts General Hospital, Boston, MA, USA

${ }^{3}$ Department of Gynecology and Obstetrics, Faculty of Health Sciences, MatCH Research Unit, University of Witwatersrand, Durban, South Africa

${ }^{4}$ Center for Global Health, Massachusetts General Hospital, Boston, Massachusetts, USA

${ }^{5}$ Division of Clinical Pharmacology, Johns Hopkins Medicine, Baltimore, Maryland, USA

${ }^{6}$ Department of Global Health, University of Washington, Seattle, Washington, USA ${ }^{7}$ Department of Global Health and Social Medicine, Harvard Medical School, Boston, Massachusetts, USA

${ }^{8}$ Department of Biostatistics, Harvard T.H. Chan School of Public Health, Boston, Massachusetts, USA

${ }^{9}$ Department of Psychiatry, Massachusetts General Hospital, Boston, MA ${ }^{10}$ Dean's Office, Portland State University School of Public Health, Oregon Health and Science University, Portland, Oregon, USA

Contributors LTM: is the PI and oversees all aspects of the work; designed the safer conception counselling component and training. DRB: helped develop the idea and the original protocol. JEH and $\mathrm{CH}$ : supported the adherence analysis plan. JMB: provided protocol input. KW: wrote the statistical analysis plan, monitors ongoing data collection, and cleans and analyses the data for 6-monthly reports to the Data Safety Monitoring Board (DSMB). JAS, YK and MJ: are implementing the trial in South Africa. YK: drafted the protocol paper. PS: revised the draft paper. KO'N: monitors data collection and helped to develop study tools. NCW: designed the qualitative study design and supported development of the qualitative tools. CP: designed and supports implementation of the adherence counseling component.

Funding This work is supported by funding from Massachusetts General Hospital (Executive Committee on Research), the NIH (NIMHK23MH095655,
NIMHR01MH108412), and the Sullivan Family Foundation. Gilead Pharmaceuticals is providing Truvada as PrEP.

Competing interests None declared.

Patient consent for publication Not required.

Provenance and peer review Not commissioned; externally peer reviewed.

Open access This is an open access article distributed in accordance with the Creative Commons Attribution Non Commercial (CC BY-NC 4.0) license, which permits others to distribute, remix, adapt, build upon this work non-commercially, and license their derivative works on different terms, provided the original work is properly cited, appropriate credit is given, any changes made indicated, and the use is non-commercial. See: http://creativecommons.org/licenses/by-nc/4.0/.

\section{REFERENCES}

1. Matthews LT, Crankshaw T, Giddy J, et al. Reproductive decisionmaking and periconception practices among HIV-positive men and women attending HIV services in Durban, South Africa. AIDS Behav 2013;17:461-70

2. Cooper D, Moodley J, Zweigenthal V, et al. Fertility intentions and reproductive health care needs of people living with HIV in Cape Town, South Africa: implications for integrating reproductive health and HIV care services. AIDS Behav 2009;13(S1):38-46.

3. Mindry D, Maman S, Chirowodza A, et al. Looking to the future: South African men and women negotiating HIV risk and relationship intimacy. Cult Health Sex 2011;13:589-602.

4. Myer L, Morroni C, Rebe K. Prevalence and determinants of fertility intentions of HIV-infected women and men receiving antiretroviral therapy in South Africa. AIDS Patient Care STDS 2007;21:278-85.

5. Thomson KA, Hughes J, Baeten JM, et al. Increased risk of HIV acquisition among women throughout pregnancy and during the postpartum period: a prospective per-coital-act analysis among women with HIV-infected partners. J Infect Dis 2018;218:16-25.

6. Human Sciences Research Council, S.A. South African national HIV prevalence, incidence, behaviour, and communication survey, 2017, 2018.

7. Vandormael A, Akullian Adam N, Dobra Adrian, et al. Sharp decline in male HIV incidence in a rural South African population (2004-2015); 4-7 March 2018, Boston, Massachusetts:CROI.

8. Dovel K, Yeatman S, Watkins S, et al. Men's heightened risk of AIDSrelated death: the legacy of gendered HIV testing and treatment strategies. AIDS 2015;29:1123-5.

9. Mambanga P, Sirwali RN, Tshitangano T. Factors contributing to men's reluctance to seek HIV counselling and testing at Primary Health Care facilities in Vhembe District of South Africa. Afr J Prim Health Care Fam Med 2016;8:e1-7.

10. Mitchell S, Cockcroft A, Lamothe G, et al. Equity in HIV testing: evidence from a cross-sectional study in ten Southern African countries. BMC Int Health Hum Rights 2010;10:23.

11. Osler M, Hilderbrand K, Goemaere E, et al. The continuing burden of advanced HIV disease over 10 years of increasing antiretroviral therapy coverage in South Africa. Clin Infect Dis 2018;66:S118-S125.

12. Huerga $\mathrm{H}$, Van Cutsem G, Ben Farhat $J$, et al. Who needs to be targeted for HIV testing and treatment in KwaZulu-Natal? Results from a population-based survey. J Acquir Immune Defic Syndr 2016;73:411-8.

13. World Health Organization. World Health Organization: PMTCT strategic vision 2010-2015: preventing mother-to-child transmission of HIV to reach the UNGASS and Millennium Development Goals. Geneva: World Health Organization, 2010.

14. Matthews LT, Beyeza-Kashesya J, Cooke I, et al. Consensus statement: supporting safer conception and pregnancy for men and women living with and affected by HIV. AIDS Behav 2018;22:1713-24.

15. Matthews LT, Baeten JM, Celum C, et al. Periconception preexposure prophylaxis to prevent HIV transmission: benefits, risks, and challenges to implementation. AIDS 2010;24:1975-82.

16. Heffron R, Pintye J, Matthews LT, et al. PrEP as peri-conception HIV prevention for women and men. Curr HIVIAIDS Rep 2016;13:131-9.

17. Mofenson LM, Baggaley RC, Mameletzis I. Tenofovir disoproxil fumarate safety for women and their infants during pregnancy and breastfeeding. AIDS 2017;31:213-32.

18. Haberer JE. Current concepts for PrEP adherence in the PrEP revolution: from clinical trials to routine practice. Curr Opin HIV AIDS 2016;11:10-17. 
19. Heffron R, Thomson K, Celum C, et al. Fertility Intentions, Pregnancy, and Use of PrEP and ART for Safer Conception Among East African HIV Serodiscordant Couples. AIDS Behav 2018;22:1758-65.

20. Ngure K, Kimemia G, Dew K, et al. Delivering safer conception services to HIV serodiscordant couples in Kenya: perspectives from healthcare providers and HIV serodiscordant couples. J Int AIDS Soc 2017;20(Suppl 1):21309.

21. Schwartz SR, Bassett J, Holmes CB, et al. Client uptake of safer conception strategies: implementation outcomes from the Sakh'umndeni Safer Conception Clinic in South Africa. J Int AIDS Soc 2017;20(Suppl 1):21291.

22. Matthews LT, Heffron R, Mugo NR, et al. High medication adherence during periconception periods among HIV-1-uninfected women participating in a clinical trial of antiretroviral pre-exposure prophylaxis. J Acquir Immune Defic Syndr 2014;67:91-7.

23. Heffron R, Ngure K, Odoyo J, et al. Pre-exposure prophylaxis for HIV-negative persons with partners living with HIV: uptake, use, and effectiveness in an open-label demonstration project in East Africa. Gates Open Res 2017;1:3.

24. Bekker LG, Roux S, Sebastien E, et al. Daily and non-daily preexposure prophylaxis in African women (HPTN 067/ADAPT Cape Town Trial): a randomised, open-label, phase 2 trial. Lancet HIV 2018;5:e68-e78.

25. Brofenbrenner U. The ecology of human development. American Psychology 1979;32:513-31.

26. van der Straten A, Stadler J, Montgomery E, et al. Women's experiences with oral and vaginal pre-exposure prophylaxis: the VOICE-C qualitative study in Johannesburg, South Africa. PLoS One 2014;9:e89118.

27. Crankshaw TL, Matthews LT, Giddy J, et al. A conceptual framework for periconception HIV transmission risk reduction among HIV serodiscordant couples. Reprod Health Matters 2012;20:50-60.

28. National Department of Health. S.A., National antenatal sentinel HIV and syphilis prevalence survey in South Africa, 2011. Pretoria: National Department of Health, 2012.

29. Bekker LG, Rebe K, Venter F, et al. Southern African guidelines on the safe use of pre-exposure prophylaxis in persons at risk of acquiring HIV-1 infection. South Afr J HIV Med 2016;17:455.

30. Matthews LT, Moore L, Crankshaw TL, et al. South Africans with recent pregnancy rarely know partner's HIV serostatus: implications for serodiscordant couples interventions. BMC Public Health 2014;14:843.

31. Bunting L, Boivin J. Development and preliminary validation of the fertility status awareness tool: FertiSTAT. Hum Reprod 2010;25:1722-33.

32. Harichund C, Haripersad K, Ramjee R. Participant verification: prevention of co-enrolment in clinical trials in South Africa. S Afr Med J 2013;103:491-3.

33. Department of Health Republic of South Africa. National contraception and fertility planning policy and service delivery guidelines. Pretoria, 2012.

34. Khidir H, Psaros C, Greener L, et al. Developing a safer conception intervention for men living with HIV in South Africa. AIDS Behav 2018;22:1725-35.

35. Matthews LT, Burns BF, Bajunirwe F, et al. Beyond HIVserodiscordance: partnership communication dynamics that affect engagement in safer conception care. PLoS One 2017;12:e0183131.

36. Bolton P, Bass J, Neugebauer R, et al. Group interpersonal psychotherapy for depression in rural Uganda: a randomized controlled trial. JAMA 2003;289:3117-24.
37. Hays RB, Rebchook GM, Kegeles SM. The Mpowerment project: community-building with young gay and bisexual men to prevent HIV1. Am J Community Psychol 2003;31:301-12.

38. Latkin CA, Knowlton AR. Micro-social structural approaches to HIV prevention: a social ecological perspective. AIDS Care 2005;17(Suppl 1):102-13.

39. Haberer JE, Bangsberg DR, Baeten JM, et al. Defining success with HIV pre-exposure prophylaxis: a prevention-effective adherence paradigm. AIDS 2015;29:1277-85.

40. U.S. Public Health Service, Centers for Disease Control and Prevention, and U.S. Department of Health and Human Services. Preexposure prophylaxis for the prevention of HIV infection in the United States - 2014, 2014. Clinical Providers' Supplement.

41. World Health Organization. Guideline on when to start antiretroviral therapy and on pre-exposure prophylaxis for HIV. Geneva: W.H.O., 2015.

42. Donnell D, Baeten JM, Bumpus NN, et al. HIV protective efficacy and correlates of tenofovir blood concentrations in a clinical trial of PrEP for HIV prevention. J Acquir Immune Defic Syndr 2014;66:340-8.

43. Hendrix CW. Exploring concentration response in HIV preexposure prophylaxis to optimize clinical care and trial design. Cell 2013;155:515-8.

44. Castillo-Mancilla JR, Searls K, Caraway P, et al. Short communication: tenofovir diphosphate in dried blood spots as an objective measure of adherence in HIV-infected women. AIDS Res Hum Retroviruses 2015;31:428-32.

45. Best BM, Burchett $\mathrm{S}$, Li H, et al. Pharmacokinetics of tenofovir during pregnancy and postpartum. HIV Med 2015;16:502-11.

46. Anderson PL, Liu AY, Castillo-Mancilla JR, et al. Intracellular Tenofovir-Diphosphate and Emtricitabine-Triphosphate in Dried Blood Spots following Directly Observed Therapy. Antimicrob Agents Chemother 2018;62.

47. Patton M, Research Q. Qualitative research and evaluation methods. 3rd edn. Thousand Oaks, CA: Sage Publications, 2002.

48. García-Lerma JG, Otten RA, Qari SH, et al. Prevention of rectal SHIV transmission in macaques by daily or intermittent prophylaxis with emtricitabine and tenofovir. PLoS Med 2008;5:e28.

49. Haberer JE, Baeten JM, Campbell J, et al. Adherence to antiretroviral prophylaxis for HIV prevention: a substudy cohort within a clinical trial of serodiscordant couples in East Africa. PLoS Med 2013;10:e1001511

50. Grant RM, Lama JR, Anderson PL, et al. Preexposure chemoprophylaxis for HIV prevention in men who have sex with men. N Engl J Med 2010;363:2587-99.

51. Abdool Karim Q, Abdool Karim SS, Frohlich JA, et al. Effectiveness and safety of tenofovir gel, an antiretroviral microbicide, for the prevention of HIV infection in women. Science 2010;329:1168-74.

52. Baeten JM, Donnell D, Ndase P, et al. Antiretroviral prophylaxis for HIV prevention in heterosexual men and women. $N$ Engl $J$ Med 2012;367:399-410.

53. Cottrell ML, Garrett KL, Prince HMA, et al. Single-dose pharmacokinetics of tenofovir alafenamide and its active metabolite in the mucosal tissues. J Antimicrob Chemother 2017;72:1731-40.

54. Little R, Rubin D. Statistical analysis with missing data. Hoboken, NJ: Wiley John \& Sons, 2002.

55. Scharfstein DO, Rotnitzky A, Robins JM. Adjusting for nonignorable drop-out using semiparametric nonresponse models. ¡ Am Stat Assoc 1999;94

56. Tsai AC, Kohrt BA, Matthews LT, et al. Promises and pitfalls of data sharing in qualitative research. Soc Sci Med 2016;169:191-8. 